\title{
COMPOSIÇÃO FLORÍSTICA DE UMA ÁREA DE CERRADO SENSU STRICTO NO MUNICÍPIO DE SENADOR MODESTINO GONÇALVES, VALE DO JEQUITINHONHA (MG) E ANÁLISE DE SIMILARIDADE FLORÍSTICA DE ALGUMAS ÁREAS DE CERRADO EM MINAS GERAIS ${ }^{1}$
}

\author{
Andreza Viana Neri² ${ }^{2}$ João Augusto Alves Meira Neto ${ }^{3,6}$, Alexandre Francisco da Silva ${ }^{3}$, Sebastião \\ Venâncio Martins ${ }^{4}$ e Amilcar Walter Saporetti Junior ${ }^{3}$
}

\begin{abstract}
RESUMO - O conhecimento da flora do Cerrado e dos fatores que influenciam a distribuição das espécies nesse bioma se faz necessário, principalmente, em razão do acelerado avanço da agricultura sob suas áreas nativas. Com o intuito de auxiliar o conhecimento da botânica, procurou-se conhecer a florística de uma área de Cerrado em Senador Modestino Gonçalves, MG, e possíveis ligações florísticas entre algumas áreas de Cerrado em Minas Gerais. Para tal, fez-se um levantamento florístico em Senador Modestino Gonçalves e uma análise de similaridade dessa comunidade com outras 27 áreas através do índice de Sfrensen. Os dendrogramas foram construídos a partir de algoritmos de médias não ponderadas (UPGMA). Foram encontradas 91 espécies distribuídas em 65 gêneros e 38 famílias. As famílias que apresentaram maior riqueza foram Leguminosae (13), Malpighiaceae (11), Myrtaceae (7), Vochysiaceae (4), Sapindaceae (4) e Rubiaceae (4). No estudo comparativo, pôde-se encontrar forte similaridade da vegetação de Cerrado no Estado de Minas Gerais, porém os grupos formados não mostraram padrões fitogeográficos. Formaram-se seis grupos que apresentaram similaridades superiores a 0,5. No entanto, não foi possível verificar quais os possíveis fatores que influenciaram a formação desses grupos, mas a proximidade geográfica e a altitude parecem influenciar fortemente alguns grupos.
\end{abstract}

Palavras-chave: Cerrado, Minas Gerais, Vale do Jequitinhonha e similaridade florística.

\section{FLORISTIC COMPOSITION OF A CERRADO SENSO STRICTU AREA IN SENADOR MODESTINO GONCALVES, VALE DO JEQUITINHONHA(MG) AND FLORISTIC SIMILARITY ANALYSIS OF SOME CERRADO AREAS IN MINAS GERAIS}

\begin{abstract}
Studies on factors influencing species distribution are necessary because of the accelerated agricultural advancement over these species. Thus, this study aimed to assess Senador Modestino Gonçalves floristic composition and possible cerradofloristic connections in Minas Gerais. Twenty-eight areas were assessed for floristic similarity, including Senador Modestino Gonçalves. Sfrensen index was used in comparative analyses. Floristic analysis identified 91 species, 65 genera and 38 families. Greater richness was represented by Leguminosae (13), Malpighiaceae (11), Myrtaceae (7), Vochysiaceae (4), Sapindaceae (4), and Rubiaceae (4). A strong floristic similarity was found in cerrado vegetation, with six groups being formed with superior similarities for 0.5. However, the factors influencing this distribution could not be verified, but geographical proximity and altitude seem to have a strong influence on some of these groups.
\end{abstract}

Keywords: Cerrado, Minas Gerais, Vale do Jequitinhonha, and floristic similarity.

\footnotetext{
${ }^{1}$ Recebido em 09.03.2006 e aceito para publicação em 27.06.2007.

${ }^{2}$ Centro Universitário do Leste de Minas Gerais, Ipatinga-MG.

${ }^{3}$ Departamento de Biologia Vegetal da Universidade Federal de Viçosa (UFV).

${ }^{4}$ Departamento de Engenharia Florestal da UFV.

${ }^{5}$ Programa de Pós-Graduação em Botânica da UFV.

${ }^{6}$ Autor para contato. E-mail: <j.meira@ufv.br>.
} 


\section{INTRODUÇÃO}

A vegetação de Cerrado cobre dois milhões de $\mathrm{km}^{2}$, representando $23 \%$ do território brasileiro. Esse é o segundo maior bioma do Brasil, superado apenas pela Floresta Amazônica, com aproximadamente 3,5 milhões de $\mathrm{km}^{2}$. O Cerrado estende-se desde áreas marginais da Floresta Amazônica a áreas do sul de São Paulo e Paraná, ocupando mais de $20^{\circ}$ de latitude e atingindo altitudes de $1.800 \mathrm{~m}$. Além do Brasil, áreas de Cerrado podem ser encontradas também na Bolívia, no Paraguai e na Venezuela (RATTER et al., 1997).

As temperaturas médias anuais no Cerrado variam de 18 a $28^{\circ} \mathrm{C}$, e a precipitação pluviométrica oscila entre 800 e 2000 mm com uma forte estação seca de variada amplitude durante o inverno (DIAS, 1996).

No Brasil, o Cerrado é constituído de cinco diferentes fitofisionomias, as quais apresentam dois extremos, uma fisionomia florestal denominada cerradão, onde há predomínio da vegetação lenhosa e uma fisionomia campestre, o campo limpo, onde, além da vegetação herbácea, se encontram também pequenos subarbustos. As demais fitofisionomias, cerrado sensu stricto, campo cerrado e campo sujo, são vegetações ecotonais entre o cerradão e o campo limpo (COUTINHO, 1978).

O solo no Cerrado na maioria das vezes é distrófico com baixa disponibilidade de nutrientes, baixo $\mathrm{pH}$ e alta concentração de alumínio. Geralmente, são solos profundos e bem drenados (LOPES e COX, 1977). Porém, os variados tipos de solo que ocorrem nesse bioma fazem que, dentro de extensa área ocupada por essa vegetação, existam regiões floristicamente distintas, definidas por espécies mais bem adaptadas às condições locais (DURIGAN et al., 2003a). Muitos outros fatores são também responsáveis pelo mosaico vegetacional no Cerrado, clima, fogo e ações antrópicas (COUTINHO, 1990; PIVELLO e COUTINHO, 1996; RATTER et al., 1997; DEZZEO et al., 2004).

Padrões fitogeográficos para o Cerrado vêm sendo estabelecidos através de estudos feitos por diferentes pesquisadores (RATTER e DARGIE, 1992; CASTRO e MARTINS, 1999; RATTER et al., 1996, 2000, 2002, 2003). Padrões regionais para o Cerrado do Estado de São Paulo também têm sido estudados (DURIGAN et al., 2003a,b), e nesse ambiente o clima parece ser o fator principal para a formação de padrões florísticos e como fator secundário o solo.
Com o avanço acelerado das monoculturas sobre a vegetação de Cerrado, estudos fitogeográficos que possam auxiliar o delineamento de políticas públicas com o intuito de conservação e manejo dos recursos ainda existentes no bioma se fazem, contudo, urgentes. Com isso, este estudo teve como principal objetivo conhecer um pouco mais a respeito da distribuição do Cerrado em Minas Gerais, procurando, assim, responder às seguintes perguntas:

A área de Cerrado estudada apresenta maior similaridade florística com as áreas geograficamente mais próximas?

O Cerrado em Minas Gerais apresenta padrão fitogeográfico?

Se ocorre um padrão fitogeográfico no Cerrado de Minas Gerais, quais os possíveis fatores responsáveis pela formação desses padrões?

\section{MATERIAL E MÉTODOS}

O Município de Senador Modestino Gonçalves localiza-se na Bacia do Rio Jequitinhonha (MG), sob as coordenadas $43^{\circ} 10^{\prime} \mathrm{W}$ a $43^{\circ} 20^{\prime} \mathrm{W}$ e $17^{\circ} 40^{\prime} \mathrm{S}$ a $17^{\circ}$ $43^{\circ} \mathrm{S}$, a cerca de $373 \mathrm{~km}$ de Belo Horizonte (Figura 1). O trabalho foi realizado em uma RPPN, isolada por talhões de eucalipto, localizada na fazenda Ticó, propriedade da Companhia Agrícola Florestal-Santa Bárbara (CAF). A área de estudo é caracterizada principalmente por cerrado sensu stricto, mas ocorrem também campo cerrado e cerradão. A região é caracterizada por relevo plano elevado, com altitude variando de $887 \mathrm{~m}$ a $949 \mathrm{~m}$; o solo da área é do tipo Latossolo Vermelho-Amarelo (EMBRAPA, 1999), precipitação média anual de 1.059 mm e temperatura média anual de $21^{\circ} \mathrm{C}$ (CAF, 2001).

A lista florística apresentada para Senador Modestino Gonçalves foi amostrada em 30 parcelas de $10 \times 20 \mathrm{~m}$, as quais foram utilizadas em estudo de estrutura da vegetação. A identificação do material botânico foi realizada com o auxílio de literatura especializada, consulta a herbários e a especialistas. Para classificação dos táxons foi utilizado o sistema de Cronquist (1981), exceto em Leguminosae, onde foi considerado o sistema de Engler (1898) apud Joly (1977).

Para a identificação da existência de um possível padrão fitogeográfico para a distribuição do Cerrado em Minas Gerais, foram usados os estudos citados na Tabela 1, totalizando 28 áreas pertencentes a 25 
municípios, incluindo Senador Modestino Gonçalves. A localização das áreas usadas para comparação florística pode ser visualizada na Figura 1, na qual o Município de Jaboticatubas se refere à área estudada na Serra do Cipó e o Município de Francisco Dumont, a área estudada na Serra do Cabral.

A partir da lista florística dos trabalhos citados anteriormente foi construída uma matriz de presença e ausência que totalizou 402 espécies. Estas foram cuidadosamente acrescentadas na análise, procurandose não incluir sinonímias, através da atualização de nomes científicos pelo site do Missouri Botanical Garden (2005).

A análise de similaridade florística entre os dados deste estudo e de 27 outros em cerrados de Minas Gerais foi calculada a partir do índice de Sfrensen (Is), obtido pela fórmula (BROWER e ZAR, 1984):

$$
\mathrm{Is}=2 \mathrm{c} / \mathrm{a}+\mathrm{b}
$$

em que:

$\mathrm{a}=$ número total de espécies do local A;

$\mathrm{b}=$ número total de espécies do local $\mathrm{B}$; e

$\mathrm{c}=$ número de espécies comuns entre o local A e B.

Para que os possíveis agrupamentos pudessem ser visualizados foram construídos dendrogramas a partir do método de algoritmos de médias não ponderadas (UPGMA) (SNEATH e SOKAL, 1973).

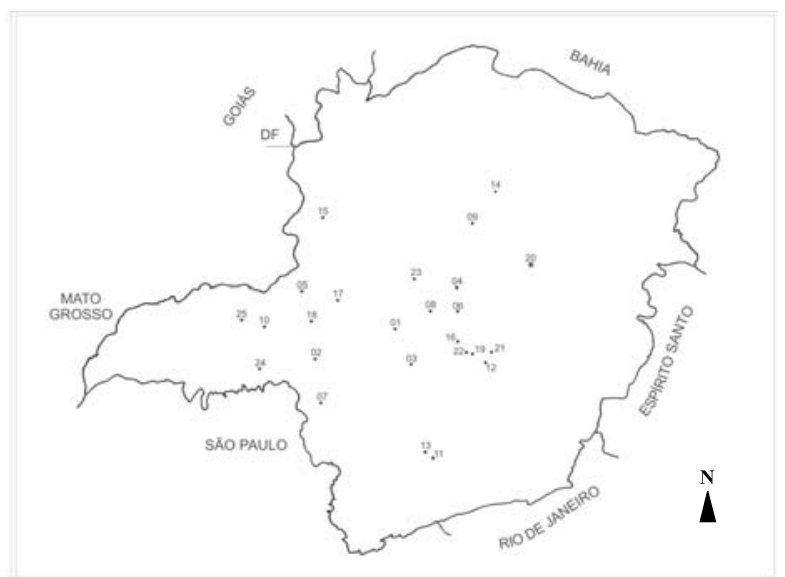

Figura 1 - Localização dos municípios onde foram realizados os trabalhos utilizados na análise de similaridade florística. O município ao qual se refere cada número está representado na tabela 1.

Figure 1-Map showing the districts where floristic similarity analyses were carried out in Minas Gerais, Brazil. The district to which each number refers is represented in Table 1.

\section{RESULTADOS E DISCUSSÃO}

\subsection{Florística}

Foram encontradas 91 espécies distribuídas em 65 gêneros e 38 famílias (Tabela 2). Das espécies encontradas, 82 foram classificadas em nível específico, oito em nível de gênero, duas em nível de família (1 Myrtaceae e 1 Sapindaceae) e uma está indeterminada. Das famílias apresentadas na Tabela 2, 37 pertencem à classe Magnoliopsida, e somente uma pertence à classe Liliopsida, estando representada pela família Arecaceae (Syagrus flexuosa). As famílias mais representativas foram Leguminosae (13), Malpighiaceae (11), Myrtaceae (7), Vochysiaceae (4), Sapindaceae (4), e Rubiaceae (4) (Tabela 2).

As famílias Leguminosae, Malpighiaceae, Myrtaceae, Vochysiaceae e Rubiaceae comumente se destacam no Cerrado, com relação à riqueza específica. Leitão-Filho (1992), em um estudo da flora arbórea de Cerrado do Estado de São Paulo, e Araújo et al. (1997), em um estudo no Município de Uberlândia, destacaram a família Myrtaceae como detentora do maior número de espécies. Felfili et al. (1998) numa análise comparativa do Cerrado sensu stricto do Brasil central, Silva et al. (2002), no Parque Estadual da Serra de Caldas Novas, GO, e Costa e Araújo (2001), na Reserva do Panga, em Uberlândia-MG, destacaram Leguminosae em função de sua alta riqueza florística.

A predominância de espécies da família Leguminosae é freqüente não só em áreas onde ocorre o Cerrado, mas, também, em outros tipos vegetacionais brasileiros. Dentro de Leguminosae, a sub família Faboideae apresenta o maior número de espécies no Cerrado, como foi constatado nos trabalhos de Goodland (1979), Araújo et al. (1997), Costa e Araújo (2001), Silva et al. (2002) e Balduíno (2005).

O solo do Cerrado é reconhecidamente deficiente em nutrientes disponíveis e possui grande concentração de alumínio livre. As plantas que ocorrem nesse ambiente possuem diversas estratégias para se desenvolverem sob altos teores de alumínio livre. Em razão das diferentes estratégias apresentadas pelas plantas do Cerrado, elas foram, então, classificadas como acumuladoras obrigatórias ou facultativas de alumínio e nãoacumuladoras tolerantes ou não-acumuladoras sensíveis ao alumínio (GOODLAND, 1979). As plantas não acumuladoras tolerantes são altamente especializadas

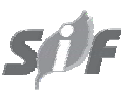

R. Árvore, Viçosa-MG, v.31, n.6, p.1109-1119, 2007 
e compreendem a maior parte das plantas de Cerrado. Pertencem principalmente às famílias Leguminosae, Malpighiaceae, Myrtaceae, Asteraceae e Dilleniaceae (GOODLAND, 1979). As três primeiras famílias citadas apresentaram-se neste trabalho como as mais ricas, confirmando a importância delas na composição florística das áreas de Cerrado.

Vochysiaceae tem sido considerada a família mais característica da vegetação de Cerrado, estando sempre entre as famílias com maior riqueza florística e, principalmente, pela considerável abundância (RIBEIRO e HARIDASAN, 1984). Ela é uma pequena família neotropical, composta de seis gêneros (GOODLAND, 1979), e destes apenas Qualea foi encontrado neste estudo. Vochysiaceae e Rubiaceae são constituídas de plantas essencialmente acumuladoras de alumínio (GOODLAND, 1979). No Cerrado, as espécies dessas famílias apresentam maior vantagem competitiva sobre as outras plantas, principalmente em áreas de solos distróficos (HARIDASAN, 2000) e álicos.

Tabela 1 - Coordenadas geográficas, altitudes e referência das áreas que foram utilizadas na comparação florística com o Cerrado senso stricto da Fazenda Tico, Município de Senador Modestino Gonçalves, Minas Gerais, Brasil. COD refere-se ao código da cidade no mapa na Figura 1

Table 1 - Geographical coordinates, altitudes and reference of the areas used for floristic similarity analysis with cerrado sensu stricto at Fazenda Ticó, Senador Modestino Gonçalves district, Minas Gerais, Brazil. COD refers to the district code in Figure 1 map

\begin{tabular}{|c|c|c|c|c|}
\hline Município & COD & Coordenadas & ALT. & Referência \\
\hline Abaeté & 1 & $19^{\circ} 09^{\prime} 36^{\prime \prime} \mathrm{S} 45^{\circ} 26^{\prime} 45^{\prime \prime} \mathrm{W}$ & $647 \mathrm{~m}$ & Saporetti Jr et al. 2003a \\
\hline Araxá & 2 & $19^{\circ} 35^{\prime} 36^{\prime \prime} \mathrm{S} 46^{\circ} 56^{\prime} 26^{\prime \prime} \mathrm{W}^{*}$ & $997 \mathrm{~m}^{*}$ & Brandão e Gavilanes, 1992. \\
\hline Bom Despacho & 3 & $19^{\circ} 44^{\prime} 11^{\prime \prime} \mathrm{S} 45^{\circ} 15^{\prime} 08^{\prime \prime} \mathrm{W}$ & $769 \mathrm{~m}$ & Saporetti Jr et al. 2003b \\
\hline Corinto & 4 & $18^{\circ} 22^{\prime} 51^{\prime \prime} \mathrm{S} 44^{\circ} 27^{\prime} 23^{\prime \prime} \mathrm{W}^{*}$ & $636 \mathrm{~m}^{*}$ & Brandão e Gavilanes, 1992. \\
\hline Coromandel & 5 & $18^{\circ} 28^{\prime} 24^{\prime \prime} \mathrm{S} 47^{\circ} 12^{\prime} 01^{\prime \prime} \mathrm{W}^{*}$ & $976 \mathrm{~m}^{*}$ & Brandão e Gavilanes, 1992. \\
\hline Curvelo & 6 & $18^{\circ} 45^{\prime} 23^{\prime \prime} \mathrm{S} 44^{\circ} 25^{\prime} 51^{\prime \prime} \mathrm{W}^{*}$ & $632 \mathrm{~m} *$ & Brandão e Gavilanes, 1992. \\
\hline Delfinópolis & 7 & $20^{\circ} 15^{\prime}-20^{\circ} 30^{\prime} \mathrm{S} 46^{\circ} 45^{\prime}-47^{\circ} 00^{\prime} \mathrm{W}$ & $684 \mathrm{~m} *$ & Bonifácio-Silva (dados não publ.) \\
\hline Felixlândia & 8 & $18^{\circ} 45^{\prime} 29^{\prime \prime} \mathrm{S} 44^{\circ} 53^{\prime} 56^{\prime \prime} \mathrm{W}^{*}$ & $614 \mathrm{~m}^{*}$ & Brandão e Gavilanes, 1992. \\
\hline Francisco Dumont & 9 & $17^{\circ} 18^{\prime} 54^{\prime \prime} \mathrm{S} 44^{\circ} 14^{\prime} 03^{\prime \prime} \mathrm{W}^{*}$ & $1000 \mathrm{~m}$ & Neri (dados não publicados) \\
\hline Indianópolis & 10 & $19^{\circ} 02^{\prime} 19^{\prime \prime} \mathrm{S} 47^{\circ} 55^{\prime} 01^{\prime \prime} \mathrm{W}$ & $809 \mathrm{~m}$ & Silva et al. (dados não publicados) \\
\hline Itumirim & 11 & $21^{\circ} 19^{\prime} 01^{\prime \prime} \mathrm{S} 44^{\circ} 52^{\prime} 16^{\prime \prime} \mathrm{W}^{*}$ & $871 \mathrm{~m}^{*}$ & Brandão e Gavilanes, 1992. \\
\hline Lagoa Santa & 12 & $19^{\circ} 37^{\prime} 38^{\prime \prime} \mathrm{S} 43^{\circ} 53^{\prime} 23^{\prime \prime} \mathrm{W}^{*}$ & $760 \mathrm{~m}^{*}$ & Brandão e Gavilanes, 1992. \\
\hline Lavras & 13 & $21^{\circ} 14^{\prime} 43^{\prime \prime} \mathrm{S} 44^{\circ} 59^{\prime} 59^{\prime \prime} \mathrm{W}^{*}$ & $919 \mathrm{~m}^{*}$ & Brandão e Gavilanes, 1992. \\
\hline Montes Claros & 14 & $16^{\circ} 44^{\prime} 06^{\prime \prime} \mathrm{S} 43^{\circ} 51^{\prime} 42^{\prime \prime} \mathrm{W}^{*}$ & $648 \mathrm{~m}^{*}$ & Brandão e Gavilanes, 1992. \\
\hline Panga -cerradão & 25 & $\begin{array}{c}19^{\circ} 09^{\prime} 20^{\prime \prime}-19^{\circ} 11^{\prime} 10^{\prime \prime} \mathrm{S} 48^{\circ} 23^{\prime} 20^{\prime \prime}- \\
48^{\circ} 24^{\prime} 35^{\prime \prime} \mathrm{W}\end{array}$ & $800 \mathrm{~m}$ & Costa e Araújo, 2001. \\
\hline Panga -cerrado & 25 & $\begin{array}{c}19^{\circ} 09^{\prime} 20^{\prime \prime}-19^{\circ} 11^{\prime} 10^{\prime \prime} \mathrm{S} 48^{\circ} 23^{\prime} 20^{\prime \prime} \\
-48^{\circ} 24^{\prime} 35^{\prime} \mathrm{W}\end{array}$ & $800 \mathrm{~m}$ & Costa e Araújo, 2001. \\
\hline Paracatu & 15 & $17^{\circ} 13^{\prime} 20^{\prime \prime} \mathrm{S} 46^{\circ} 52^{\prime} 29^{\prime \prime} \mathrm{W}^{*}$ & $900 \mathrm{~m}$ & Felfili et al., 1993. \\
\hline Paraopeba & 16 & $19^{\circ} 20^{\prime} \mathrm{S} 44^{\circ} 20^{\prime} \mathrm{W}$ & $740 \mathrm{~m}$ & Bauduino, 2005. \\
\hline Paraopeba & 16 & $19^{\circ} 20^{\prime} \mathrm{S} 44^{\circ} 20^{\prime} \mathrm{W}$ & $740 \mathrm{~m}$ & Brandão e Gavilanes, 1992. \\
\hline Patos de Minas & 17 & $18^{\circ} 34^{\prime} 44^{\prime \prime} \mathrm{S} 46^{\circ} 31^{\prime} 05^{\prime \prime} \mathrm{W}^{*}$ & $832 \mathrm{~m}^{*}$ & Brandão e Gavilanes, 1992. \\
\hline Patrocínio & 18 & $18^{\circ} 56^{\prime} 38^{\prime \prime} \mathrm{S} 46^{\circ} 59^{\prime} 33^{\prime \prime} \mathrm{W}^{*}$ & $950 \mathrm{~m}$ & Felfili et al., 1993. \\
\hline Prudente de Morais & 19 & $19^{\circ} 28^{\prime} 55^{\prime \prime} \mathrm{S} 4^{\circ} 09^{\prime} 18^{\prime \prime} \mathrm{W}^{*}$ & $748 \mathrm{~m}^{*}$ & Brandão e Gavilanes, 1992. \\
\hline Senador Modestino & 20 & $43^{\circ} 10^{\prime} \mathrm{W}-43^{\circ} 19^{\prime} \mathrm{W} 17^{\circ} 42^{\prime} \mathrm{S}-17^{\circ} 49^{\circ} \mathrm{S}$ & $918 \mathrm{~m}$ & Presente Estudo \\
\hline \multicolumn{5}{|l|}{ Gonçalves } \\
\hline Serra do Cipó & 21 & $19^{\circ} 22^{\prime} 01 ' \mathrm{~S} 43^{\circ} 37^{\prime} 10^{\prime \prime} \mathrm{W}$ & $850 \mathrm{~m}^{*}$ & Meira Neto e Saporetti-Jr, 2002. \\
\hline Sete Lagoas & 22 & $19^{\circ} 27^{\prime} 57^{\prime \prime} \mathrm{S} 44^{\circ} 14^{\prime} 48^{\prime \prime} \mathrm{W}^{*}$ & $761 \mathrm{~m}^{*}$ & Brandão e Gavilanes, 1992. \\
\hline Três Marias & 23 & $18^{\circ} 12^{\prime} 23^{\prime \prime} \mathrm{S} 45^{\circ} 14^{\prime} 30^{\prime \prime} \mathrm{W}^{*}$ & $538 \mathrm{~m}^{*}$ & Brandão e Gavilanes, 1992. \\
\hline Uberaba & 24 & $19^{\circ} 44^{\prime} 54^{\prime \prime} \mathrm{S} 47^{\circ} 55^{\prime} 55^{\prime \prime} \mathrm{W}^{*}$ & $801 \mathrm{~m}^{*}$ & Brandão e Gavilanes, 1992. \\
\hline Uberlândia & 25 & $18^{\circ} 55^{\prime} 23^{\prime \prime} \mathrm{S} 48^{\circ} 17^{\prime} 19^{\prime \prime} \mathrm{W}$ & $863 \mathrm{~m} *$ & Araújo et al., 1997. \\
\hline
\end{tabular}

*Informações obtidas do site www.aondefica.com/vxhjlu.asp.

R. Árvore, Viçosa-MG, v.31, n.6, p.1109-1119, 2007 
Tabela 2 - Lista das espécies encontradas no levantamento florístico de uma área de Ccerrado sensu stricto no Município de Senador Modestino Gonçalves, MG, Brazil. A lista encontra-se em ordem alfabética de família, gênero e espécie

Table 2 -Species found in the floristic assessment of a cerrado sensu stricto area of Senador Modestino Gonçalves, Minas Gerais, Brazil. The list is in alphabetic order by family, genera and species

Família/Espécie

1 Anacardiaceae

Tapirira guianensis Aubl.

2 Annonaceae

Annona dioica A. St.-Hil.

Duguetia furfuracea (A. St.-Hil.) Benth. \& Hook. f.

3 Apocynaceae

Aspidosperma macrocarpon Mart.

Aspidosperma tomentosum Mart.

Himatanthus obovatus (Müll. Arg.) Woodson

4 Araliaceae

Didymopanax macrocarpum Seem.

Didymopanax morototoni (Aubl.) Decne. \& Planch.

5 Arecaceae

Syagrus flexuosa (Mart.) Becc.

6 Asteraceae

Gochnatia pulchra Cabrera

Lychnophora salicifolia Mart..

Vernonia sp.

7 Bignoniaceae

Cybistax antisyphilitica (Mart.) Mart.

Tabebuia ochracea (Cham.) Standl.

Zeyheria digitalis (Vell.) L. B. Sm. Sandwith

8 Bombacaceae

Eriotheca pubescens (Mart. \& Zucc.) Schott \& Endl.

9 Burseraceae

Protium heptaphyllum (Aubl.) Marchand

10 Caryocaraceae

Caryocar brasiliense Cambess.

11 Celastraceae

Austroplenckia populnea (Reissek) Lundell

12 Connaraceae

Rourea induta Planch.

13 Dilleniaceae

Davilla rugosa Poir.

14 Ebenaceae

Diospyros hispida A. DC.

Diospyros sericea A. DC.

15 Erythroxylaceae

Erythroxylum daphnites Mart.

Erythroxylum tortuosum Mart.

Erythroxylum gonocladum (C. Mart.) O. E. Schulz

16 Euphorbiaceae Pera glabrata (Schott) Poepp. ex Baill.
Tabela 2 - Cont.

Table 2 - Cont.

Família/Espécie

17 Flacourtiaceae

Casearia sylvestris $\mathrm{Sw}$.

18 Guttifera

Kielmeyera petiolaris Mart.

Kielmeyera coriacea Mart.

19 Lauraceae

$$
\text { Ocotea felix Coe - Teixeira }
$$

20 Leguminosae

Acosmium dasycarpum (Vogel) Yakovlev

Bauhinia rufa (Bong.) Steud.

Bowdichia virgilioides Kunth

Dalbergia brasiliensis Vogel

Dalbergia miscolobium Benth.

Enterolobium gummiferum (Mart.) J. F. Macbr.

Hymenaea stigonocarpa Mart. ex Hayne

Machaerium opacum Vogel

Mimosa verrucosa Benth.

Plathymenia reticulata Benth.

Sclerolobium paniculatum Vogel

Senna rugosa (G. Don) H.S. Irwin \& Barneby

Stryphnodendron adstringens (Mart.) Coville

21 Loganiaceae

Strychnos pseudoquina A. St.-Hil.

22 Lythraceae

Lafoensia pacari A. St.-Hil.

23 Malpighiaceae

Banisteriopsis cf anisandra (A. Juss.) B. Gates

Banisteriopsis campestris A. Juss.

Banisteriopsis malifolia (Nees \& Mart.) B. Gates

Banisteriopsis stellaris (Griseb.) B. Gates

Byrsonima coccolobaefolia Kunth

Byrsonima intermedia A. Juss.

Byrsonima salzmanniana A. Juss.

Byrsonima verbascifolia (L.) DC.

Byrsonima sp.

Heteropterys byrsonimifolia A. Juss

Heteropterys eglandulosa A. Juss

24 Melastomataceae

Miconia albicans (Sw.) Triana

Miconia ligustroide (DC.) Naudin

25 Meliaceae

Cabralea cangerana Saldanha

26 Moraceae

Brosimum gaudichaudii Trécul

27 Myrsinaceae

Rapanea guianensis Aubl.

28 Myrtaceae

Eugenia aurata O. Berg

Eugenia dysenterica DC.

Eugenia sp.

$$
\text { Continua ... }
$$$$
\text { Continued... }
$$

Myrcia multiflora (Lam.) DC.

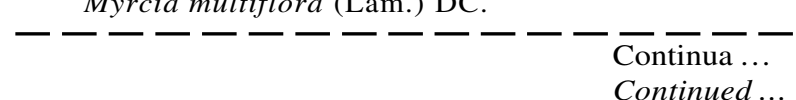

R. Árvore, Viçosa-MG, v.31, n.6, p.1109-1119, 2007 
Tabela 2 - Cont.

Table 2 - Cont.

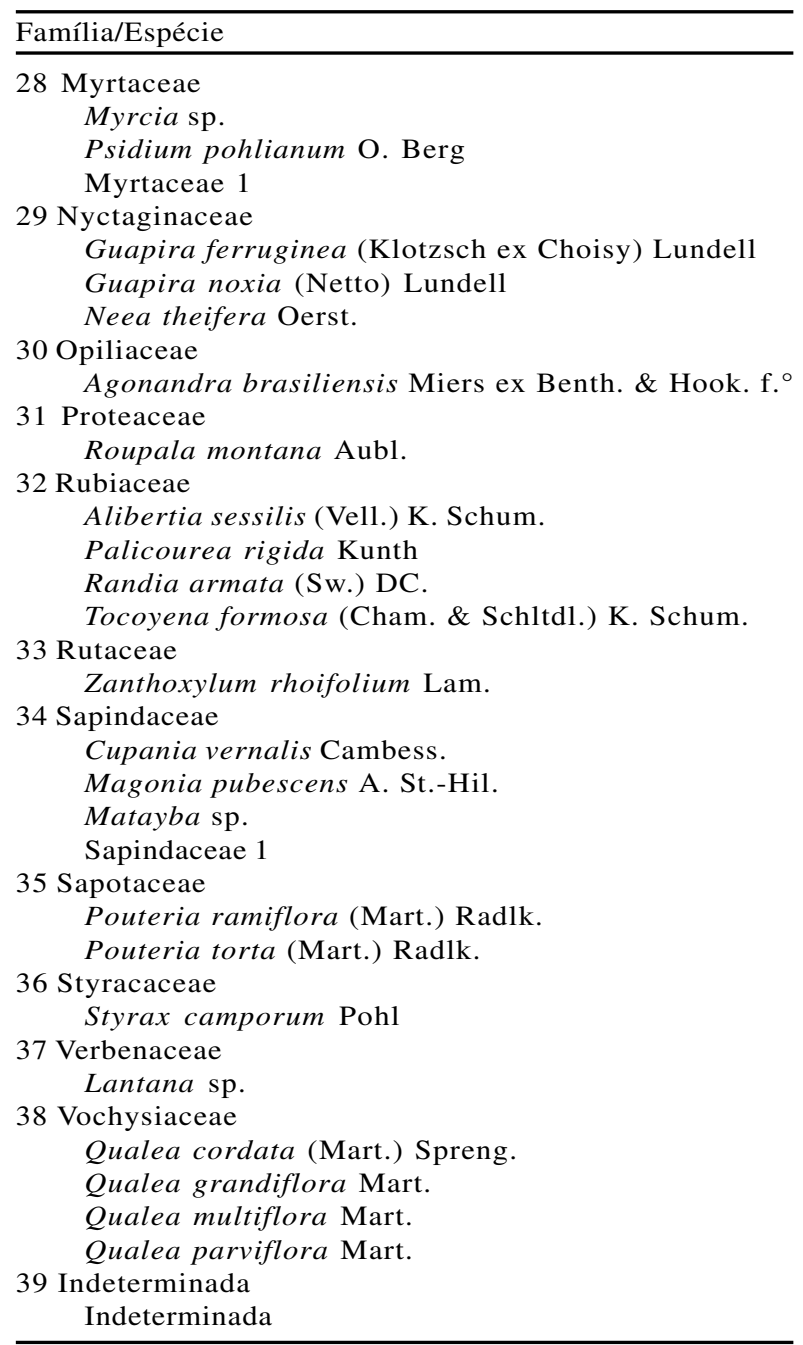

Malpighiaceae se destacou neste estudo, assim como em outros em áreas de Cerrado (FELFILI et al., 1993; ARAÚJO et al., 1997; MUNHOZ e PROENÇA, 1998; COSTA e ARAÚJO, 2001; BATALHA e MANTOVANI, 2001; ANDRADE et al., 2002), em função do gênero Byrsonima, que apresentou a maior riqueza específica. Além do gênero Byrsonima (5), outro gênero que também apresentou alta riqueza específica foi Banisteriopsis (4).

O gênero Byrsonima é um importante componente da vegetação de Cerrado. Ele foi encontrado por Goodland (1979) em todos os 110 locais estudados no Triângulo Mineiro, MG, sendo predominante em alguns deles. Esse gênero também se destaca quanto à riqueza específica em outros trabalhos, como na Chapada da Pratinha (FELFILI et al., 1993), em cerrados residuais do Município de Uberlândia (ARAÚJO et al., 1997) e em áreas de Cerrado sensu stricto e cerradão da Reserva do Panga, Uberlândia, MG (COSTA e ARAÚJO, 2001). No trabalho de Rizzini (1963), esse gênero foi representado por 10 espécies, já em um trabalho complementar ao do Rizzini a riqueza deste foi ainda maior, 22 espécies (HERINGER et al., 1977).

Neste estudo, mereceu destaque também o alto número de espécies, principalmente, quando comparado com os trabalhos utilizados na análise de similaridade florística. Na Reserva do Panga, Costa e Araújo (2001) encontraram em área de cerradão 93 espécies (1,0 ha) e em área de Cerrado sensu stricto 76 espécies $(0,68$ ha). Em 1,0 ha amostrado num Cerrado sensu stricto em Paraopeba, MG, Balduíno et al. (2005) encontraram 74 espécies, Saporetti Jr. et al. (2003b) encontraram 39 espécies em Bom Despacho, MG (0,3 ha), e Saporetti Jr. et al. (2003a) 87 espécies em Abaeté, MG (0,3 ha).

Em áreas muito estudadas como o Distrito Federal, o Estado de São Paulo e parte do sul de Minas Gerais, a flora do Cerrado é bem conhecida e é pouco provável que ocorra espécies novas no componente lenhoso. Porém, em áreas como norte do Mato Grosso e norte de Minas Gerais, essa probabilidade é maior (CASTRO et al., 1999), em função do menor número de trabalhos. Dessa forma, é necessário que esforços sejam realizados no intuito de conhecer essas áreas, antes que sejam completamente desmatadas.

\subsection{Similaridade florística}

Através da análise comparativa das 28 áreas de cerrado de Minas Gerais (Figura 2), puderam-se observar grupos com forte similaridade florística. A análise apontou uma definição clara de seis grupos bem consistentes com similaridades superiores a 0,5 .

O grupo 1 foi formado principalmente por áreas localizadas no norte e centro de Minas Gerais, nos Municípios de Curvelo, Corinto, Três Marias, Felixlândia, Montes Claros, Prudente de Morais, Sete Lagoas, Lagoa Santa e Paraopeba (1992), com uma similaridade de 0,68. Esse grupo compartilhou 37 espécies. As regiões que compõem o grupo 1 são caracterizadas pela menores altitudes, variando de $538 \mathrm{~m}$ a $761 \mathrm{~m}$. A baixa altitude dessas áreas pode ter proporcionado condições ambientais semelhantes, sendo, portanto, a altitude nesse caso uma característica muito importante para 
o agrupamento florístico dessas áreas. Esse grupo parece ser formado por espécies que possuem ampla distribuição, visto que não apresentou nenhuma espécie exclusiva. As principais espécies responsáveis pelo agrupamento das áreas são as denominadas espécies de ligação; elas aparecem exclusivamente em combinações de duas amostras. Na formação do grupo 1, as espécies Astronium fraxinifolium, Dictyoloma incanescens, Guazuma ulmifolia e Schynus terebinthifolius são espécies de ligação exclusivas desse grupo.
O grupo 2 composto por áreas localizadas ao sul do Estado, em Lavras e Itumirim, possui em comum 40 espécies que lhe conferiu uma similaridade de 0,63. A alta similaridade provavelmente se deve à proximidade das duas áreas. Esse grupo apresentou apenas uma espécie exclusiva, Machaerium villosum. Das espécies de ligação desse grupo, Bauhinia forficata, Cordia trichotoma, Jacaranda caroba, Luehea paniculata, Machaerium villosum, Miconia theezans e Ouratea castanaefolia não aparecem como espécies de ligação de outros grupos.

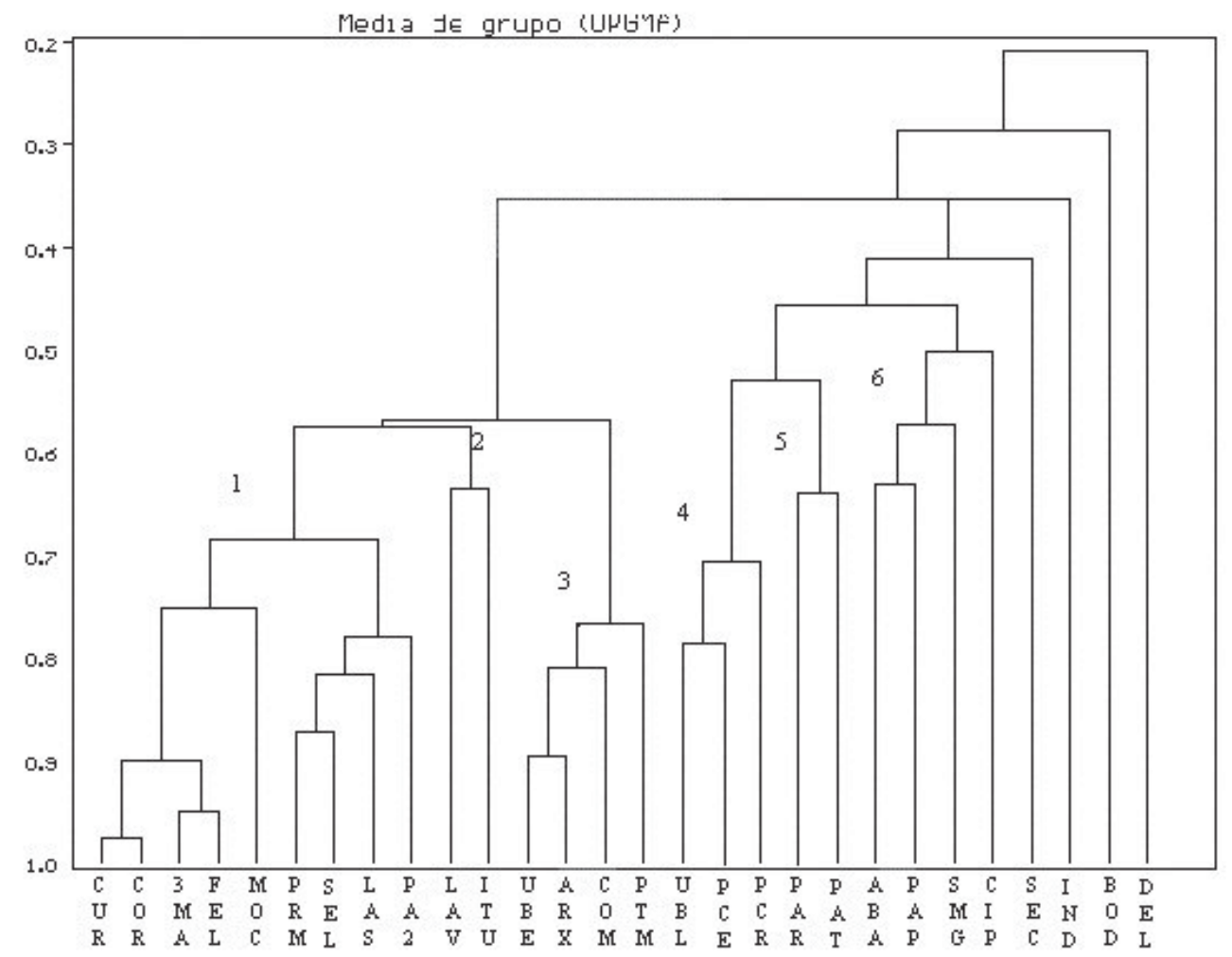

Figura 2 - Dendrograma gerado a partir do índice de similaridade de Sfrensen pelo método de aglomeração de algoritmos de médias não ponderadas (UPGMA) das 28 áreas de cerrado do Estado de Minas Gerais, utilizando-se a flora lenhosa. CUR (Curvelo), COR (Corinto), 3MA (Três Marias), FEL (Felixlândia), MOC (Montes Claros), PRM (Prudente de Morais), SEL (Sete Lagoas), LAS (Lagoa Santa), PA2 (Paraopeba, 1992), LAV (Lavras), ITU (Itumirim), UBE (Uberaba), ARX (Araxá), COM (Coromandel), PTM (Patos de Minas), UBL (Uberlândia), PCE (Panga Cerrado), PCR (Panga Cerradão), PAR (Paracatu), PAT (Patrocínio), ABA (Abaeté), PAP (Paraopeba-2001), SMG (Senador Modestino Gonçalves), CIP (Cipó), SEC (Serra do Cabral), IND (Indianópolis), BOD (Bom Despacho) e DEL (Delfinópolis). Citações na Tabela 1.

Figure 2 -Dendrogram from Sfrensen similarity index by the nonweighted pair-groups method using arithmetic averages (UPGMA) of the 28 cerrado areas of the state of Minas Gerais, Brazil, using woody flora. CUR (Curvelo), COR (Corinto), 3MA (Três Marias), FEL (Felixlândia), MOC (Montes Claros), PRM (Prudente de Morais), SEL (Sete Lagoas), LAS (Lagoa Santa), PA2 (Paraopeba, 1992), LAV(Lavras), ITU (Itumirim), UBE (Uberaba), ARX (Araxá), COM (Coromandel), PTM (Patos de Minas), UBL (Uberlândia), PCE (Panga cerrado), PCR (Panga cerradão), PAR (Paracatu), PAT (Patrocínio), ABA (Abaeté), PAP (Paraopeba-2001), SMG (Senador Modestino Gonçalves), CIP (Cipó), SEC (Serra do Cabral), IND (Indianópolis), BOD (Bom Despacho), DEL (Delfinópolis). References are shown in Table 1. 
O grupo 3, composto por áreas do Triângulo Mineiro localizadas em Uberaba, Araxá, Coromandel e Patos de Minas, compartilha 31 espécies. Esse grupo apresentou alta similaridade, 0,78. Das espécies que uniram esse grupo, Miconia pepericarpa, Casearia arborea, Luehea divaricata e Tapirira guianensis não são espécies de ligação, de outros grupos. A espécie Miconia pepericarpa, além de ser espécie de ligação aparece apenas nas áreas do grupo 3.

O grupo 4 também é composto por áreas do Triângulo Mineiro, porém sua ligação com o grupo 3 é baixa, em relação à ligação deste com os grupos 1 e 2. As três áreas do grupo 4 ficam no Município de Uberlândia; a primeira (UBL) representa uma lista florística de um trabalho em que foram amostradas 20 áreas de cerrados residuais nesse município que foram incluídas nesta análise como apenas uma amostragem, a segunda (PCE) e a terceira (PCR) são áreas de Cerrado sensu stricto e cerradão da Reserva do Panga. A proximidade dessas áreas, que provavelmente possuem as mesmas condições ambientais (precipitação e mesmo período de seca), é que devem ter-lhes conferido alta similaridade florística $(0,70)$.

$\mathrm{O}$ alto grau de similaridade alcançado entre as áreas do grupo 4, que apresentou 55 espécies em comum, inclui desde espécies de ampla distribuição no Cerrado até espécies com distribuições mais restritas. Vatairea macrocarpa, Andira paniculata, Cardiopetalum calophyllum, Dalbergia elliptica e Guapira graciliflora apresentaram-se nessa análise como espécies exclusivas desse grupo. As espécies Acosmium subelegans, Andira paniculata, Austroplenckia populnea, Byrsonima basiloba, Cardiopetalum calophyllum, Davilla elliptica, Eriotheca gracilipes Guapira graciliflora, Licania humilis, Matayba guianensis, Myrcia rostrata, Myrcia variabilis, Plathymenia reticulata, Pouteria ramiflora, Pouteria torta, Rudgea viburnoides, Sclerolobium aureum e Tapirira guianensis são espécies de ligação desse grupo e que não aparecem como espécies de ligação de outros grupos.

O grupo 5, composto por duas áreas, Patrocínio e Paracatu, obteve uma similaridade florística de 0,64. Essas áreas não apresentaram espécies exclusivas, mas possuem 33 espécies em comum. Essas áreas por estarem próximas do Brasil central, provavelmente recebem grandes influências tanto da vegetação de Cerrado quanto das florestas adjacentes dessa região. Em um trabalho de comparação entre Cerrado sensu stricto nas Chapadas Pratinha e Veadeiros (FELFILI et al., 1997), essas duas regiões também mostraram grande similaridade florística. Esses autores ainda destacaram a importância da criação de Unidades de Conservação em Paracatu e Patrocínio, por se tratar de áreas muito dissimilares das demais estudadas (FELFILI et al., 1997, 2001). Annona coriacea, Aspidosperma tomentosum, Casearia sylvestris, Connarus suberosus, Eremanthus glomerulatus, Erythroxylum deciduum, Heteropteris byrsonimaefolia, Pera glabrata, Tabebuia caraiba, Terminalia argentea e Vochysia elliptica são espécies de ligação exclusivas do grupo 5 .

O grupo 6 foi formado com uma similaridade florística de 0,57 pelas áreas de Abaeté, Paraopeba (2001) e Senador Modestino Gonçalves. Esse grupo não apresentou espécies restritas e foi o grupo que apresentou o menor número de espécies de ligação; destas, Alibertia sessilis, Enterolobium gummiferum, Erythroxylum daphnites, Palicourea rigida, Protium heptaphyllum, Sclerolobium paniculatum e Syagrus flexuosa são espécies de ligação do grupo 6 que não aparecem como espécies de ligação de outros grupos. A proximidade geográfica não justifica a similaridade desse grupo, como foi questionado nos objetivos. Esse é um grupo que se dispõe como um corredor, e provavelmente outro fator seja responsável por essa ligação florística.

Considerando valores menores de similaridade, puderam-se observar três grandes grupos, o primeiro formado pelos grupos 1, 2 e 3, que apresentaram grande similaridade florística de 0,57. Essas áreas exibiram variadas altitudes e regiões com diferentes condições climáticas, possuindo provavelmente um outro fator responsável pela elevada similaridade florística. $\mathrm{O}$ segundo grande grupo formado pelos grupos 4 e 5 , com similaridade de 0,53 , provavelmente é onde a altitude exerça maior influência, já que nesse grupo houve variação muito pequena com relação a esse fator (800 m a $950 \mathrm{~m}$ ). O terceiro, formado pelo grupo 6 e a área estudada na Serra do Cipó, apresentou a menor similaridade $(0,50)$. Ao contrário do segundo grupo, esse último apresentou maior variação de altitudes (647 $\mathrm{m}$ a $918 \mathrm{~m})$.

As áreas de Indianópolis, Delfinópolis, Bom Despacho e Serra do Cabral foram as que se mostraram menos similares. Aárea amostrada na Serra do Cabral 
foi a que apresentou a maior altitude, além de ser uma área altamente impactada. Delfinópolis provavelmente se mostrou pouco similar às outras áreas, por ser um estudo de florística e apresentar grande número de espécies.

Ratter et al. (1996), analisando 98 áreas de Cerrado distribuídas em todo o território nacional, encontraram um padrão de distribuição fitogeográfica formado por seis grupos, que foram classificados de acordo com a localidade, um grupo ao sul, um grupo a sudeste, um grupo central, um grupo no centro-oeste, um grupo ao norte e um grupo formado pelo cerrado encravado na Amazônia. Nesse trabalho, o clima e, particularmente, a precipitação e o tempo da estação seca foram fatores importantes para a distribuição da vegetação do Cerrado, assim como o efeito da altitude. Os fatores ambientais citados por esses autores provavelmente também são responsáveis pela formação dos diferentes grupos florísticos apresentados no Estado de Minas Gerais.

Neste estudo, entretanto, não foi possível saber ao certo quais poderiam ser os fatores responsáveis pela similaridade dos grupos formados. A maioria dos trabalhos avaliados não continha informações ambientais suficientes para uma discussão mais detalhada. Mas foi possível perceber que altitude e proximidade geográfica são fatores importantes na similaridade de algumas áreas, como os grupos 2 e 4.

No grupo 6 onde ficou inserida a área de Senador Modestino Gonçalves não foi possível inferir qual o possível fator responsável pela similaridade florística do grupo, já que altitude e proximidade geográfica não parecem ser.

Com o número de áreas utilizadas em estudo de similaridade, o Cerrado de Minas Gerais não mostrou possuir um padrão fitogeográfico, mas a forte ligação florística entre os grupos demonstrou uma estreita ligação florística, mesmo entre grupos geograficamente distantes. Faz-se, então, necessária, a continuidade de estudos dessa natureza para que se possam investigar fatores bióticos ou abióticos responsáveis por essas ligações florísticas.

Além do exposto, o conhecimento da riqueza florística dos Cerrados de Minas Gerais ainda depende de mais estudos, especialmente na região norte do Estado. Isso é coerente, já que são poucos os trabalhos nessa região, e áreas como a de Senador Modestino Gonçalves, que apresentou alta riqueza específica, podem ser ainda desconhecidas. Além da florística, são necessários estudos que procurem conhecer os principais fatores responsáveis pela distribuição de algumas espécies e pela manutenção das suas fitofisionomias nesse bioma.

\section{AGRADECIMENTOS}

À Fundação de Amaro à Pesquisa do Estado de Minas Gerais (FAPEMIG) e à CAF-Santa Bárbara, pelo apoio financeiro; e aos funcionários da CAF-Santa Bárbara no Município de Carbonita, MG, pela hospitalidade e pela ajuda no trabalho de campo; também aos pareceristas anônimos, que muito contribuíram para o enriquecimento do texto.

\section{REFERÊNCIAS}

ANDRADE, L. A. Z.; FELFILI, J. M.; VIOLLATI, L. Fitossociologia de uma área de cerrado denso na RECOR-IBGE, Brasília-DF. Acta Botanica Brasílica, v.16, n.2, p.225-240, 2002.

ARAÚJO, G. M. et al. Estrutura comunitária de vinte áreas de cerrados residuais no município de Uberlândia, MG. Daphne, v.7, n.2, p.7-14, 1997.

BALDUÍNO, A P. C. et al. Fitossociologia e análise comparativa de composição florística do Cerrado na Floana de Paraopeba-MG. Revista Árvore, v.29, n.1, p.25-34, 2005.

BATALHA, M. A.; MANTOVANI, W. Floristic composition of the cerrado in the Pé-de-Gigante reserva (Santa Rita do Passa Quatro, southeastern Brazil). Acta Botanica Brasílica, v. 15, n.3, p.289-304, 2001

BONIFÁCIO-SILVA, A. C. Levantamento florístico de cinco áreas de Delfinópolis, Minas Gerais - Brasil. 2001. 80f. Dissertação (Mestrado em Ciências) Universidade de São Paulo, Ribeirão Preto, 2001.

BRANDÃO, M.; GAVILANES, M. L. Espécies arbóreas padronizadas do cerrado mineiro e sua distribuição no Estado. Informe

Agropecuário, v.16, n.173, p.5-11, 1992.

BROWER, J. E.; ZAR, J. H. Field and laboratory methods for general ecology. 2.ed. Dubuque: Wm. C. Brown, 1984. 255p.

COMPANHIA AGRÍCOLA FLORESTAL SANTA BÁRBARA - CAF. Região de Carbonita. 8.ed. Belo Horizonte: 2001.

R. Árvore, Viçosa-MG, v.31, n.6, p.1109-1119, 2007 
CASTRO, A. A. J. F.; MARTINS, F. R. Cerrados do Brasil e do Nordeste: caracterização, área de ocupação e considerações sobre a sua fitodiversidade. Pesquisa em Foco, v.7, n.9, p.147-178, 1999 .

COSTA, A. A.; ARAUJO, G. M. Comparação da vegetação arbórea de cerradão e de cerrado na Reserva do Panga, Uberlândia, Minas Gerais. Acta Botanica Brasílica, v.15, n.1, p.63-72, 2001.

COUTINHO, L. M. O conceito de cerrado. Revista Brasileira de Botânica, v.1, p.17-23, 1978.

COUTINHO, L. M. Fire in the ecology of the Brazilian cerrado. In: GOLDAMMER, J. G. (Ed.). Fire in the Tropical Biota: Ecosystem processes and global challenges. Berlin: SpringerVerlag, 1990. p. 82-105.

CRONQUIST, A. An integrated system of classification of flowering plants. New York: Columbia University, 1981. 1262p.

DEZZEO, N. et al. Changes in soil properties and vegetation characteristics along a forest-savanna gradient in southern Venezuela. Forest Ecology and Management, v.200, p.183-193, 2004.

DIAS, B. F. S. (Ed.) Alternativas de desenvolvimento dos cerrados: manejo e conservação dos recursos naturais não renováveis. 2.ed. Brasília: Fundação Pró-Natureza, 1996. 97p.

DURIGAN, G. et al. Padrões fitogeográficos do cerrado paulista sob uma perspectiva regional. Hoehnea, v.30, n.1, p.39-51, 2003a.

DURIGAN, G. et al. The vegetation of priority áreas for cerrado conservation in São Paulo state, Brazil. Edinburgh Journal of Botany, v.60, n.2, p.217-241, 2003b.

\section{EMPRESA BRASILEIRA DE PESQUISA} AGROPECUÁRIA - EMBRAPA. Centro Nacional de Pesquisa de Solos. Sistema brasileiro de classificação de solos. Brasília: 1999.412p.

FELFILI, J. M. et al. Análise comparativa da florística e fitossociologia do vegetação arbórea do cerrado sensu stricto na Chapada Pratinha, DF-Brasil. Acta Botanica Brasílica, v.6, n.2, p.27-46, 1993.

R. Árvore, Viçosa-MG, v.31, n.6, p.1109-1119, 2007
FELFILI, J. M. et al. Comparação do Cerrado (Stricto Sensu) nas Chapadas Pratinha e dos Veadeiros. In: CONGRESSO DE ECOLOGIA DO BRASIL, 3., Brasília, 1997. Anais...Brasília: Universidade de Brasília, 1996. p.6-11.

FELFILI, J. M. et al. Comparison of cerrado (sensu stricto) vegetation in central Brazil. Ciência e Cultura, v.50, n.4, p.237-243, 1998.

FELFILI, J. M.; SEVILHA, A. C.; SILVA-JÚNIOR, M. C. Comparação entre unidades fisiográficas Chapada Pratinha, Veadeiros e Espigão Mestre do São Francisco. In: FELFILI, J. M.; SILVA-JÚNIOR, M. C. (Orgs.). Biogeografia do bioma cerrado: estudo fitofisionômico na Chapada do Espigão Mestre do São Francisco. Brasília: Universidade de Brasília, 2001. p 80-90.

GOODLAND, R. Ecologia do Cerrado. Belo Horizonte: Itatiaia, 1979. 193p.

HARIDASAN, M. Nutrição mineral das plantas nativas do Cerrado - grupos funcionais. In: CAVALCANTI, T. B.; WALTER, B. M. T. (Org.). Tópicos atuais em botânica: palestras convidadas. CONGRESSO NACIONAL DE BOTÂNICA, 51., 2000, Brasília. Anais... Brasília: Embrapa Recursos Genéticos e Biotecnologia/Sociedade Botânica do Brasil, 2000. p.159-164.

HERINGER, E. P. et al. A flora do Cerrado. In: FERRI, M. G. (Ed.). SIMPÓSIO SOBRE O CERRADO, 4., 1977, São Paulo. Anais... São Paulo: Universidade de São Paulo, 1977. p.303-316

http://www.aondefica.com/vxhjlu.asp) Acesso em dezembro de 2002.

JOLY, A. B. Botânica: introdução à taxonomia vegetal. São Paulo: Nacional, 1977. 634p.

LEITÃO FILHO, H. F. A flora arbórea dos cerrados do Estado de São Paulo. Hoehnea, v.19, p.151-163, 1992.

LOPES, A. S.; COX, F. R. A survey of the fertility status of surface soils under 'cerrado' vegetation in Brazil. Soil Science Society of America Journal, v.41, p.741-747, 1977.

MEIRA NETO, J. A. A. et al. Estrutura de uma Floresta Estacional Semidecidual Aluvial em área diretamente afetada pela Usina Hidrelétrica de Pilar, Ponte Nova, Zona da Mata de Minas Gerais. Revista Árvore, v.21, n.2, p.213-219, 1997. 
MEIRA NETO, J. A. A.; SAPORETTI-JÚNIOR, A. W. Composição florística em cerrado no Parque Nacional da Serra do Cipó, MG. Revista Árvore, v.26, n.5, p.645-648, 2002.

MISSOURI BOTANICAL GARDEN. Disponível em: (http://mobot.mobot.org/W3T/Search/vast.html) Acesso em: julho de 2005.

MUNHOZ, C. B. R.; PROENÇA, C. E. B. Composição Florística do município de Alto Paraíso de Goiás na Chapada dos Veadeiros. Boletim do Herbário Ezechias Paulo Heringer, v.3, p.102-150, 1998.

NERI, A. V. Levantamento florístico e aspectos estruturais de uma área de cerrado típico, na Serra do Cabral, Francisco Dumont, MG. 2000. Monografia (Graduação em Biologia) - Universidade Estadual de Montes Claros, Montes Claros, 2000.

PIVELLO, V. R.; COUTINHO, L. M. A qualitative successional model to asssist in the management of Brazilian cerrados. Forest Ecology and Management, v.87, p.127-138, 1996.

RATTER, J. A.; DARGIE, T. C. D. An analysis of the floristic composition of 26 cerrado areas in Brazil. Edinburgh Journal of Botany, v.49, p.235-250, 1992.

RATTER, J. A. et al. Analysis of the floristic composition of the Brazilian Cerrado Vegetation II: Comparison of the woody vegetation of 98 areas. Edinburgh Journal of Botany, v.53, n.2, p.153-180, 1996.

RATTER, J. A.; RIBEIRO, J. F.; BRIDGEWATER, $S$. The Brazilian cerrado vegetation and threats to its biodiversity. Annals of Botany, v.80, p.223-230, 1997.

RATTER, J. A.; RIBEIRO, J. F.; BRIDGEWATER, $S$. Woody flora distribution of the cerrado biome: phytogeography and conservation priorities. In: CAVALCANTI, T. B. et al. (Org). CONGRESSO NACIONAL DE BOTÂNICA, 51, 2000, Brasília. Anais... Brasília: Embrapa Recursos Genéticos e Biotecnologia, Sociedade Botânica do Brasil. 2000. p. 340-342.
RATTER, J. A.; BRIDGEWATER, S.; RIBEIRO, J. F. Espécies lenhosas da fitofisionomia cerrado sentido amplo em 170 localidades do Bioma Cerrado. Boletim do Herbário Ezechias Paulo Heringer, v.5, p.5-43, 2002.

RATTER, J. A., BRIDGEWATER, S.; RIBEIRO, J. F. Analysis of the floristic compositionof the Brasilian Cerrado vegetation. III: comparison of the woody vagetation of 376 areas. Edinburgh Journal of Botany, v.60, n.1, p.57-109, 2003.

RIBEIRO, J. F.; HARIDASAN, M. Comparação fitossociológica de um cerrado denso e um cerradão em solos distróficos no Distrito Federal. In: CONGRESSO NACIONAL DE BOTÂNICA,35., 1984, Manaus. Anais...Manaus: Sociedade de Botânica do Brasil, 1984. p.342-347.

RIZZINI, C. T. A flora do Cerrado: análise florística das savanas centrais. In: SIMPÓSIO SOBRE O CERRADO. São Paulo: Universidade de São Paulo, 1963. p.125-177

RIZZINI, C. T. Tratado de Fitogeografia do Brasil. São Paulo: Universidade de São Paulo, 1979.v.2

SAPORETTI JR, A. W.; MEIRA NETO, J. A. A.; ALMADO, R. P. Fitossociologia de cerrado sensu strito no município de Abaeté, MG. Revista Árvore, v.27, n.3, p.413-419, 2003a.

SAPORETTI JR, A. W.; MEIRA NETO, J. A. A.; ALMADO, R. Fitossociologia de sub-bosque de cerrado em talhão de Eucalyptus grandis W. Hill ex Maiden no município de Bom Despacho, MG. Revista Árvore, v.27, n.6, p.905-910, 2003 b.

SILVA, A. F. et al. Estudos florísticos e fitossociológicos das áreas de influência e diretamente afetada da Usina Hidrelétrica de Miranda. LOCAL: CEMIG/SIF, 1994 v.2 (Relatório Final).

SILVA, L. O. et al. Levantamento florístico e fitossociológico em duas áreas de cerrado sensu stricto no Parque Estadual da Serra de Caldas Novas, Goiás. Acta Botanica Brasílica, v.16 n.1, p.43-53, 2002.

SNEATH, P. H. \& SOKAL, R. R. Numerical taxonomy. New York: W. H. Freeman and Company, 1973.

R. Árvore, Viçosa-MG, v.31, n.6, p.1109-1119, 2007 
